\title{
Development of a finite element model for the simulation of parabolic impact of sandwich panels
}

\author{
Karthik Ram Ramakrishnan ${ }^{1,2, a}$, Sandra Guérard ${ }^{1, b}$, Laurent Mahéo ${ }^{3}$, Krishna Shankar², and Philippe Viot $^{1}$ \\ ${ }^{1}$ Arts et Métiers ParisTech, I2M, UMR 5295, 33400 Talence, France \\ ${ }^{2}$ School of Engineering and Information Technology, UNSW, Canberra 2600, Australia \\ ${ }^{3}$ LIMATB, Université de Bretagne Sud, 56321 Lorient Cedex, France
}

\begin{abstract}
Sandwich panels are lightweight structures of two thin high strength facesheets bonded to either side of a thick low density core such as foams and honeycombs. It is necessary to study the impact response of sandwich structures in order to ensure the reliability and safety of these structures. The response of sandwich panels to impact loading is usually studied for impact at normal angle of incidence. In real engineering situations, the structures are more frequently loaded at some oblique angle or with a complex trajectory. It is easy to carry out normal impact tests using devices like the drop tower, but impacts at oblique angles are difficult to characterise experimentally. A tri-dimensional impact device called Hexapod has been developed to experimentally study the impact loading of sandwich plates with a parabolic trajectory. The Hexapod is a modified Gough-Stewart platform that can be moved independently in the six degrees of freedom, corresponding to three translation axes and three rotation axes. In this paper, an approach for modelling the parabolic impact of sandwich structures with thin metallic facesheets and polymer foam core using commercial finite element code LS-DYNA software is presented. The results of the FE model of sandwich panels are compared with experimental data in terms of the time history of vertical and horizontal components of force. A comparison of the strain history obtained from Digital Image Correlation and LS-Dyna model are also presented.
\end{abstract}

\section{Introduction}

Sandwich panels are a special form of laminated composites comprising of two thin high strength facesheets bonded to either side of a thick low density core such as foams and honeycombs. This provides a lightweight structure with high bending stiffness. The skins are designed to resist tensile and compressive stresses and are usually made of aluminium, polymers or fibre reinforced polymers. The core is designed to resist compression and shear stresses and a range of materials including end grain Balsa wood, rigid polymer foams, aluminium or Nomex honeycombs are used as cores. The standard test method for the study of low velocity impact response of sandwich panels are for normal incidence impact, i.e. the impact loading is applied along one fixed direction that is perpendicular to the plane of the target plate. Test devices like drop tower are designed for normal impact condition and the response of the panels are analysed in terms of peak load, absorbed energy and deflection at peak load. However, normal impacts rarely occur in real engineering situations and structures are more frequently loaded at some oblique angle or complex trajectory. While normal incidence impact tests are easy to carry out using devices such as the drop tower, oblique tests are difficult to undertake experimentally, given the need to guide the projectile, coupled with the presence of a horizontal force component that applies a load to the required guide rails. Zhou et al. [1] found that the impact energy for an oblique

a e-mail: karthik.ramakrishnan@adfa.edu.au

b e-mail: sandra.guerard@ensam.eu impact of sandwich composite is dissipated in shearing both the composite and the foam around the perimeter of the projectile, as well as crushing the foam ahead of the impactor. The perforation energy was found to increase with increasing angle of incidence. Goswami et al. [2] found that the debonding along the facesheets and the core interface of sandwich panels is predominated by a sliding mode and observed that low relative density of the core will result in more critical debonding [2]. Zhou et al. [1] also observed that the shear fracture properties of the foams had a significant effect on the perforation resistance of the sandwich structures, suggesting that this mode of failure is important in determining the impact resistance of sandwich structures. Chai and Zhu [3] reviewed the existing literature on the low velocity impact of sandwich structures and found that studies on sandwich panel subjected to low velocity impact at angles other than perpendicular to the plane of the sandwich were limited. Guérard et al. [4] noted that normal impacts do not correspond to the complex loading that real structures encounter and developed a tri-dimensional impact device called Hexapod to experimentally study the impact loading of sandwich plates with a parabolic trajectory. Sandwich plates with metallic facesheets and polymeric foam core were investigated.

Experiments are time consuming and expensive, and predicting the response of sandwich plates is complicated due to effects such as material and geometric nonlinearities, transverse shear effects and multiple and coupled damage modes [5]. These problems and cost of experimentation can be avoided by using numerical methods, 

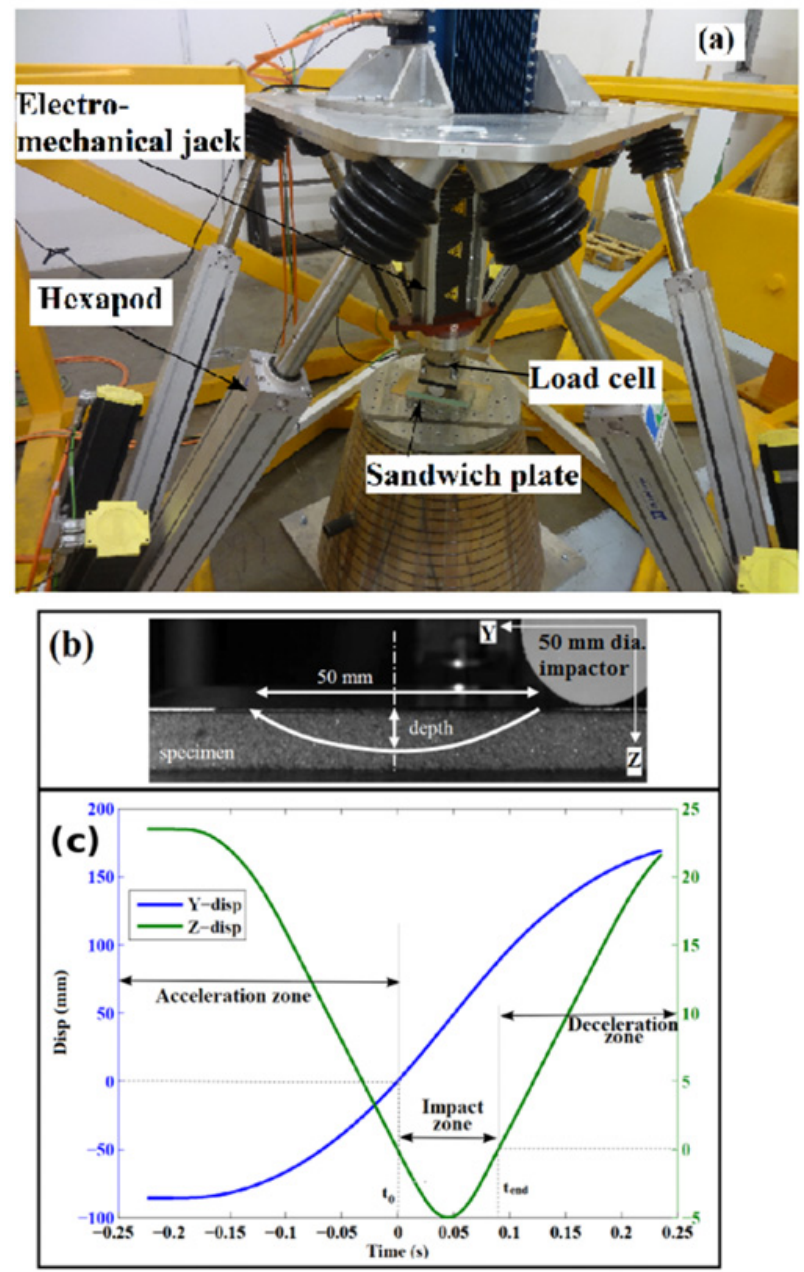

Figure 1. (a) Hexapod setup for tri-dimensional impact, (b) Parabolic trajectory and (c) Displacement input for the Hexapod.

which can help in predicting the energy absorption and peak loads by considering competing mechanisms [6-8]. The combination of improved constitutive modelling and decreased computational costs make it possible to utilise continuum modelling of sandwich composites to numerically simulate impact response for a much wider range of impactor geometries and velocities [9]. In this paper, a Finite Element model is presented for the simulation of the parabolic impact of sandwich plates.

\section{Experimental setup}

Low-velocity impact tests were performed using the Hexapod facility available in the Dynamics platform of DuMAS Department of I2M laboratory in Arts et Métiers Paristech, Bordeaux. The Hexapod, which is a modified Gough-Stewart platform, a type of parallel robot constituted of a fixed and a moving platform is shown in Fig. 1. The top plate can be moved independently in the six degrees of freedom, corresponding to three translation axes and three rotation axes. Its horizontal velocity can reach $1.4 \mathrm{~m} / \mathrm{s}$ and the maximal vertical velocity is $1 \mathrm{~m} / \mathrm{s}$. A seventh electromechanical jack (of maximum velocity
$3.5 \mathrm{~m} / \mathrm{s}$ ) is implemented on the top plate to increase the vertical speed. A cylindrical impactor is attached to the seventh jack and the sandwich sample is placed on a rigid platform. The velocity achieved with this configuration of Hexapod is equivalent to the drop tower but with the advantage of imposing a 3D trajectory to the projectile [4]. The Hexapod is setup in such a way that it is possible to change the impactor easily with other geometries such as wedge-shaped impactor.

The Hexapod is instrumented with a tri-axial load cell (Kistler, 9377C, Fx and Fy range: 3000 N, Fz range: $5000 \mathrm{~N}$ ) which is placed between the cylindrical impactor and the bottom end of the seventh jack. Load history of each impact event was measured by the 3D load cell located between the impactor and the jack extremity. A high-speed camera (Photron Fastcam APX RS, 5000 frames per second) was used to film the impact. The side of the tested sample was coated with a fine spray of paint to enhance the random texture of the foam and allow for Digital Image Correlation (DIC) calculation (VIC-2D, Correlated Solution).

The Hexapod setup was used for three dimensional impact testing of sandwich samples made of Aluminium facesheets and PVC closed-cell foam (Divinycell) core by Guérard et al. [4]. Sandwich samples with PVC foam core of thickness $20 \mathrm{~mm}$ and 2024 aluminium facesheets of two different thicknesses: $0.5 \mathrm{~mm}$ and $1 \mathrm{~mm}$ were considered. The sandwich panels were assembled using a two-component epoxy adhesive (Araldite AW 106R/hardener HV 953U) which was chosen for their high shear and peel strength. The dimensions of the sandwich samples were $200 \mathrm{~mm}$ long and $120 \mathrm{~mm}$ wide.

In the case of a parabolic impact, a trajectory is imposed to the impactor in terms of displacement curves in the Y- and Z-axis, corresponding to the horizontal and vertical directions. This is in contrast to the normal impact testing using a drop tower, where the impactor is raised to a particular height and released and the potential energy of the impactor is converted to kinetic energy. The parameters defining the parabolic impact are the depth of penetration into the impacted specimen and the width or opening of the parabola. A parabolic displacement shown in Fig. 1b was imposed to the cylindrical impactor with a depth of penetration of $5 \mathrm{~mm}$ into the impacted specimen and the width of the parabola was $50 \mathrm{~mm}$. Figure 1c also shows the translation displacement in the Y-direction and Z-direction, which is input to the hexapod. This represents the entire trajectory input to the impactor with the beginning of the contact with the sandwich target represented by $t_{0}$ and the end of contact denoted by $t_{\text {end }}$. The horizontal velocity during impact (along Y direction) was constant and equal to $1 \mathrm{~m} / \mathrm{s}$ and the vertical acceleration was equal to $4 \mathrm{~m} / \mathrm{s}^{2}$.

\section{Finite element model of sandwich plate}

A numerical model was developed using the explicit finite element software, LS-Dyna. An LS-Dyna model, shown in the Fig. 2, was created to simulate the parabolic impact of the aluminium - Divinycell sandwich. The aluminium 

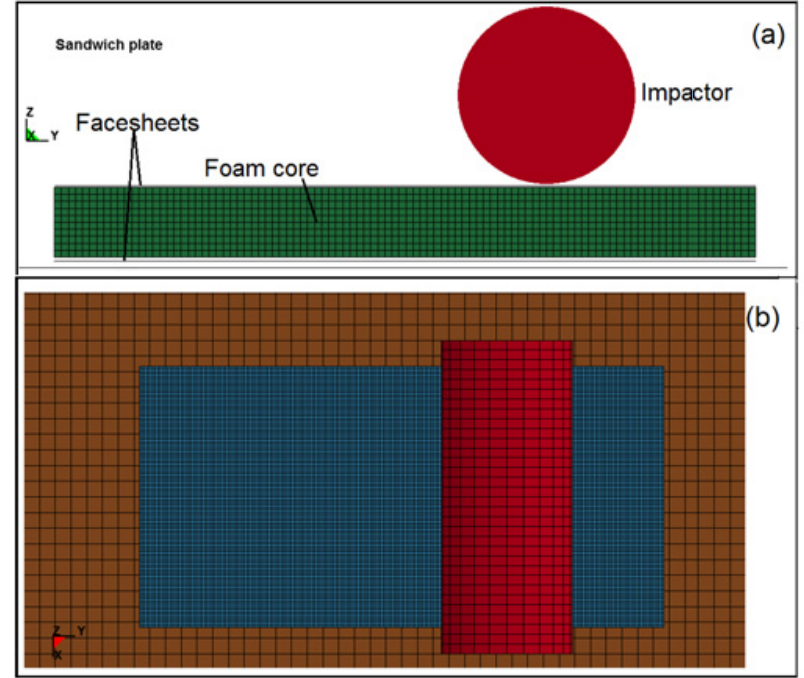

Figure 2. LS-Dyna model of Aluminium - Divinycell sandwich and cylindrical impactor.

- Divinycell sandwich plate was modelled with solid elements for the core and shell elements for the thin facesheets. Two types of sandwich plates were modelled, with $0.5 \mathrm{~mm}$ thick facesheet and $1 \mathrm{~mm}$ thick facesheet. The aluminium facesheets were modelled using BelytschkoTsai shell elements and the foam core was modelled using constant stress brick elements.

Foams are a type of cellular solids which can be described as a collection of regular or irregular thinwalled cells. The macroscopic compressive behaviour is determined by the compressive behaviour of individual cells and the collective interactions between neighbouring cells [10]. The simulation of the impact behaviour of sandwich composites is usually more complex than that of monolithic laminates. Feng and Aymerich [11] found that the mechanical properties of the core material has a strong influence on the response of the the facesheets. The failure mechanisms may also be significantly different from those developing in conventional laminates. Therefore, an accurate FE analysis of a sandwich structures requires including complex material models capable of capturing the mechanical behaviour of the core as well as the facesheets.

LS-Dyna offers a variety of material models, each with capabilities designed to capture the unique behaviour of different types of foam, for instance, open cell foams and closed cell foams. The material model chosen for the PVC foam core was MAT CRUSHABLE FOAM available in the material library of LS-Dyna as MAT63. The modelling of the foam core behaviour requires the use of models to reproduce the crushing behaviour of these cellular materials. Croop and Lobo [12] described that irrespective of the type of the foam, there are generally three zones in the compressive stress-strain relationship of foam, namely, initial elastic region up to yield, plateau compaction region and a densification region. The stressstrain response of the Divinycell foam obtained from uniaxial compression tests using Zwick electromechanical testing machine was input to the material model as

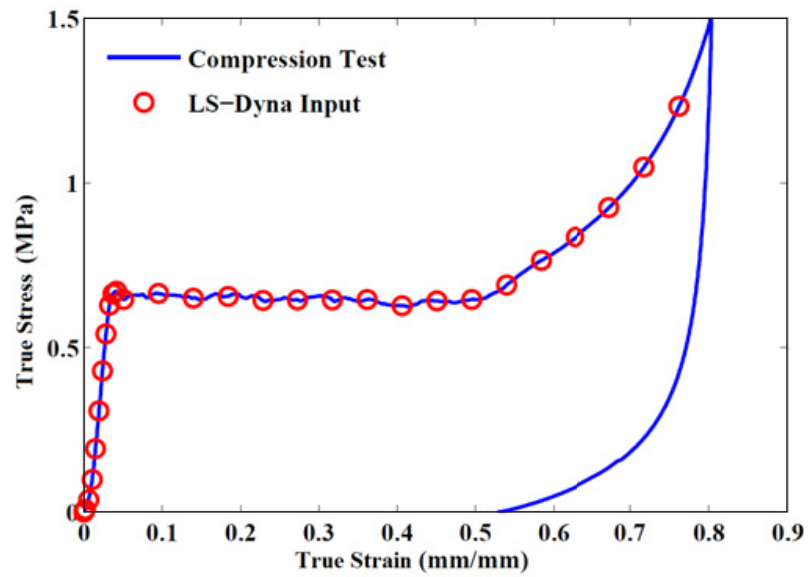

Figure 3. Material input for Divinycell foam from uniaxial compression test.

shown in the Fig. 3. The aluminium alloy facesheets were modelled with a PLASTIC KINEMATIC material law and the steel impactor was modelled with MAT RIGID. The input parameters of these materials were obtained from manufacturer datasheets.

The steel cylindrical impactor was given displacement input in the $\mathrm{Y}$ and $\mathrm{Z}$ direction by defining a curve to BOUNDARY PRESCRIBED MOTION keyword. This is in contrast to the normal impact simulation where an initial velocity input is given to the impactor. The rotation motion of the cylindrical impactor was constrained. The simulation was initiated just before the contact to reduce the run-time, with the impactor placed $0.1 \mathrm{~mm}$ above the facesheet. The total duration of the simulation is 90 milliseconds corresponding to the impact duration observed in the experiment.

The progression of core effective strain for parabolic impact with cylindrical impactor is shown in Fig. 4. It can be seen that initially when the impactor comes into contact with the sandwich plate, the strain is limited to a small region of the sandwich plate at Time $=0.01 \mathrm{~s}$ and this region grows with increasing contact duration. The strain contour for contact time of 26, 45, and 86 milliseconds are also shown in the figure. These times correspond to the point of maximum force, maximum displacement and end of contact, respectively. It can be seen that there is a strain component that is ahead of the impactor as the core elements undergo deformation.

The LS-Dyna model is validated by comparing the force-time histories with experimental data presented by Guérard et al. [4]. A comparison of the vertical and horizontal component of forces obtained from the LS-Dyna model and the experiments for the sample with $0.5-\mathrm{mm}$-thick facesheet are shown in Fig. 5a. The experimental force values are in solid lines and the FE results are in dash lines. It can be seen that the maximum vertical force (Z-force) from the experiment compares well with the simulation but the maximum horizontal force (Y-force) obtained from the simulation is much lower than the experiment. Guérard et al. [4] reported that the peak of the Z-force $(2544 \mathrm{~N})$ occurs before the maximum penetration depth whereas the peak of Y-force $(1850 \mathrm{~N})$ 


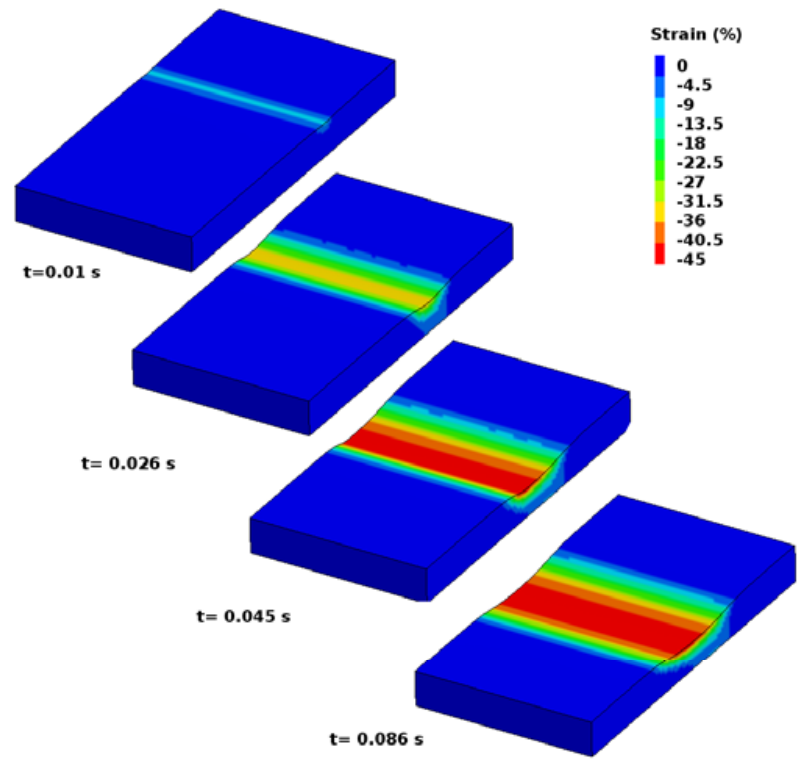

Figure 4. Progression of the impact on sandwich sample with $1 \mathrm{~mm}$ thick $\mathrm{Al}$ facesheet.

occurs slightly after which was attributed to the formation of a small wrinkle in front of the impactor during the test which generates a resistant horizontal force. The Yforce is higher in the experiment compared to the LS-Dyna model for the same reason. There is no wrinkling in the simulation and hence the value of Y-force is lower.

For the sample with $1 \mathrm{~mm}$ thick facesheet, shown in Fig. 5b, the maximum vertical force obtained from the simulation is comparable to the experiment. It can be observed that the Z-force is slightly higher for thicker aluminium facesheet with a peak of $2780 \mathrm{~N}$. The Y-force, with a peak of $1670 \mathrm{~N}$ is lower for the $1 \mathrm{~mm}$ thick facesheet compared to the $0.5 \mathrm{~mm}$ thick facesheet. This is explained by the fact that there is no observable wrinkling with the $1 \mathrm{~mm}$ thick facesheet. The horizontal component of force is under-estimated which suggests that the modelling of shear response can be improved by using a foam material model which is capable of simulating multi-axial loading.

It is also observed that the contact duration is shorter for the LS-Dyna model in both cases. One of the possible causes for this phenomenon is the permanent deformation observed in the foam material ahead of the point of contact. However, the overall characteristics of the response of the sandwich plate to parabolic impact was simulated and it can be concluded from these simulations that an LS-Dyna model can be used for the numerical modelling of tridimensional impacts.

A comparison of the strain contour of the sandwich panel is shown in Fig. 6. The effective strain obtained from Digital Image Correlation (DIC) of the high speed camera images using Vic2D software is compared with the strain contour obtained from LS-Dyna simulation for the case of the sandwich plate with $1 \mathrm{~mm}$ thick aluminium facesheets. The maximum strain at the point of maximum penetration, i.e. at 45 milliseconds, is found to be $37.8 \%$, while the corresponding strain obtained from LS-Dyna simulation is $32.98 \%$. There is large localised strain immediately below the impactor and there is permanent plastic deformation in
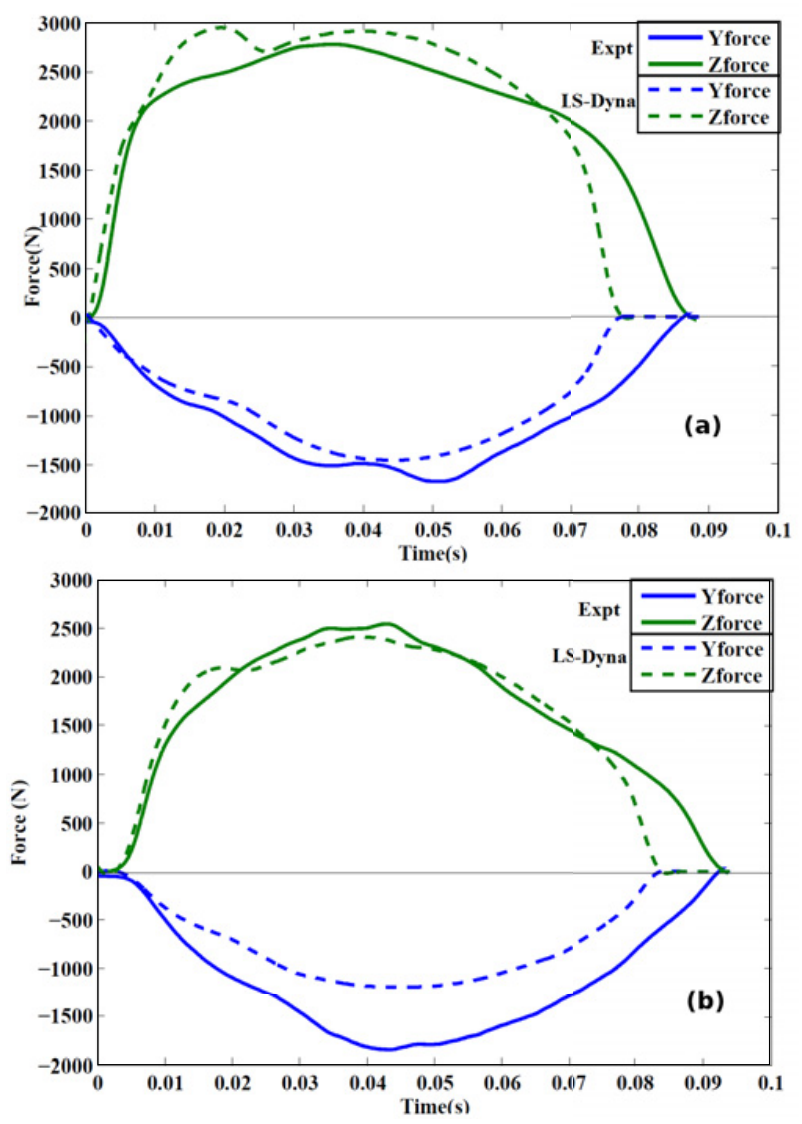

Figure 5. Force-time results obtained for parabolic impact of Aluminium Divinycell sandwich plates (a) $0.5 \mathrm{~mm}$ thick facesheet and (b) $1 \mathrm{~mm}$ thick facesheet.

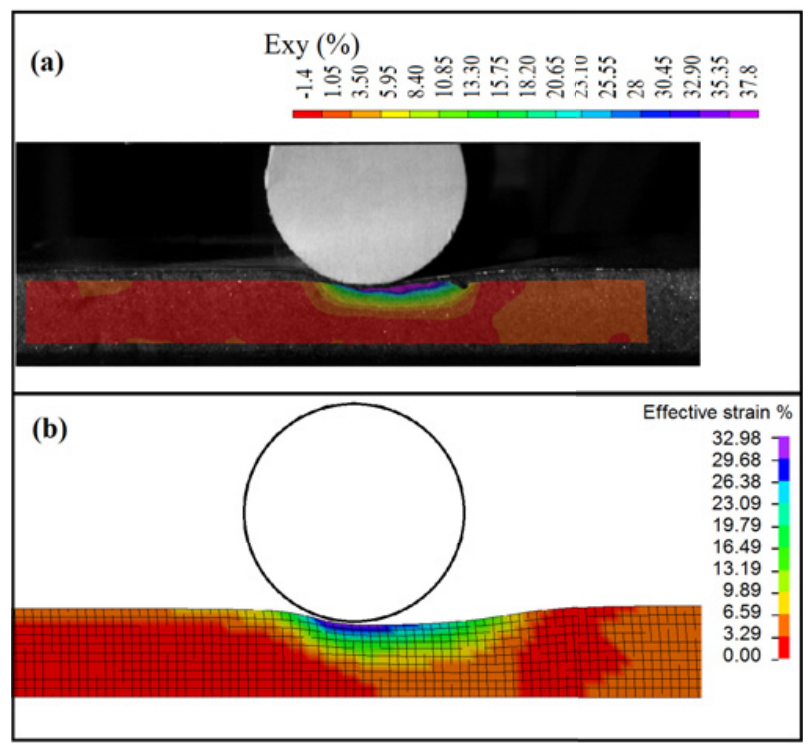

Figure 6. Effective strain of Aluminium Divinycell sandwich plates (a) DIC and (b) LS-Dyna.

both the top facesheet and the core. This is different to the normal impact case, where there is elastic recovery of the top facesheet and core crushing in the foam, which results in debonding between the core and facesheet. 


\section{Conclusion}

A new method to conduct low velocity tri-dimensional impact tests on sandwich panels using a Hexapod with parabolic trajectory of the impactor was presented. An LSDyna model was developed to reproduce the response of the sandwich panels made with Aluminium facesheets of two different thickness and PVC foam core. A crushable foam material model was used for the simulation of the Divinycell foam and bilinear kinematic model was used for the aluminium facesheets. The results of the numerical model were compared with experimental data presented by Guérard et al. [4] in terms of macroscopic force response and strain contour from digital image correlation. The peak force and maximum strain from the FE model show good correlation with experimental data. The LSDyna model provides reasonable results for the parabolic impact but more work is required to improve the model, particularly shear behaviour of foam and failure modes like debonding.

\section{References}

[1] J. Zhou, M.Z. Hassan, Z. Guan, and W.J. Cantwell. The low velocity impact response of foam-based sandwich panels. Composites Science and Technology, 72(14):1781-1790, 2012.

[2] S. Goswami and W. Becker. The effect of facesheet/core delamination in sandwich structures under transverse loading. Composite Structures, 54(4):515-521, 2001.

[3] G.B. Chai and S. Zhu. A review of low- velocity impact on sandwich structures. Proceedings of the Institution of Mechanical Engineers, Part L: Journal of Materials Design and Applications, 225(4): 207-230, 2011.
[4] S. Guérard, J-L. Barou, L. Mahéo, and P. Viot. Development of a new experimental device for tridimensional impacts - results on foam-core sandwich panels. In 4th International conference on impact loading of lightweight structures, 2014.

[5] J. Mackerle. Finite element analyses of sandwich structures: a bibliography 1980-2001. Engineering Computations, 19(2):206-245, 2002.

[6] R.C. Batra, G. Gopinath, and J.Q. Zheng. Damage and failure in low energy impact of fiber-reinforced polymeric composite laminates. Composite Structures, 94(2):540-547, 2012.

[7] C. Bouvet, S. Rivallant, and J.J. Barrau. Low velocity impact modeling in composite laminates capturing permanent indentation. Composites Science and Technology, 72(16):1977-1988, 2012.

[8] A. Rajaneesh, I. Sridhar, and S. Rajendran. Impact modeling of foam cored sandwich plates with ductile or brittle faceplates. Composite Structures, 94(5):1745-1754, 2012.

[9] J.A. Nemes and K.E. Simmonds. Low-velocity impact response of foam-core sandwich composites. Journal of Composite Materials, 26(4):500-519, 1992.

[10] E.A. Flores-Johnson and Q.M. Li. Experimental study of the indentation of sandwich panels with carbon fibre-reinforced polymer face sheets and polymeric foam core. Composites Part B: Engineering, 42(5):1212-1219, 2011.

[11] D. Feng and F. Aymerich. Damage prediction in composite sandwich panels subjected to low-velocity impact. Composites Part A: Applied Science and Manufacturing, 52(0):12-22, 2013.

[12] B. Croop and H. Lobo. Selecting material models for the simulation of foams in 1s-dyna. In 7th European LS-DYNA Conference, 2009. 